\title{
Local refinement of ERBS curves
}

\author{
Rune Dalmo \\ Narvik University College \\ Po. box. 385, N-8505 Narvik, Norway
}

\begin{abstract}
Expo-rational B-splines (ERBS) provide a blending type construction where local functions at each knot are blended together by infinitely smooth basis functions. In this work we consider some specific ERBS curves that are approximations of parametric curves. We study local refinement to increase flexibility by inserting local control curves at points of interest on the ERBS curve.

Inserting knots into an existing B-spline knot vector results in a new spline space which contains the original spline space as a sub-space. In contrast to B-splines, knot insertion with ERBS results in a rational local function. We investigate methods to generate local curves of different brands. Using this, we blend extra local curves with the original ERBS curve, by knot insertion, and compare the differences with respect to geometric shape and approximation errors.
\end{abstract}

Keywords: ERBS, approximation, interpolation, local refinement, knot insertion

PACS: 02.40.-k, 02.60.Ed

\section{INTRODUCTION}

A number of different approaches to local refinement of B-splines has been explored in previous works. We mention here knot insertion, approached differently by Boehm [1] and Cohen, Lyche and Riesenfeld [2], T-splines [3], LR-splines [4], subdivision of various kinds (e.g. the recursive method by Catmull and Clark in [5]) and macro element spaces [6]. Most of these techniques are applicable to expo-rational B-splines (ERBS)[7, 8]. However, the ERBS blending construction, using local functions as coefficients, are different from the well-known B-spline type blending construction. To the best of our knowledge, applying local refinement to ERBS has not been studied in detail yet.

Since knot insertion seems to have played an important role for establishing B-splines and NURBS as common tools in applications for CAGD, we will focus on this particular topic in this preliminary study.

\section{PRELIMINARIES}

\subsection{Expo-rational B-splines}

Let $\mathbf{t}=\left\{t_{k}\right\}_{k=0}^{n+1}$ be a strictly increasing knot vector. The Expo-rational B-Spline (ERBS) associated with $t_{k-1}, t_{k}$ and $t_{k+1}$ is defined in [7,8]. We consider here the 
scalable subset, proposed in [9], where the integrals are independent of the knot vector:

$$
B_{k}(t)= \begin{cases}S_{k-1} \int_{0}^{\omega_{k-1}(t)} \psi_{k-1}(s) d s, & t_{k-1}<t \leq t_{k}, \\ S_{k} \int_{\omega_{k}(t)}^{1} \psi_{k}(s) d s, & t_{k}<t<t_{k+1} \\ 0, & \text { otherwise }\end{cases}
$$

where $\omega_{k}(t)=\frac{t-t_{k}}{t_{k+1}-t_{k}}, \quad \psi(s)=e^{-\beta \frac{|s-\lambda|(1+\gamma) \alpha}{\left(s(1-s)^{\gamma}\right)^{\alpha}}}$, and the scaling factor is $S_{k}=$ $\left(\int_{0}^{1} \psi(s) d s\right)^{-1}$, where $\alpha>0, \beta>0, \gamma>0,0 \leq \lambda \leq 1$. The ERBS shares three of its five basic properties with the linear B-spline; partition of unity, minimal support and that it interpolates its coefficient in its central knot. In addition, all of its derivatives are zero in every knot, and it is $C^{\infty}$-smooth on $\mathbb{R}$.

We note here that the ERBS index number indicates the central knot, where it interpolates its local function (the peak of the basis function), contrary to the B-spline index number which indicates at which knot the B-spline "starts".

An ERBS function $f(t)$ is a blending type construction where local functions are interpolated at each knot. It is defined on $\left(t_{1}, t_{n}\right]$ by

$$
f(t)=\sum_{k=1}^{n} \ell_{k}(t) B_{k}(t), t \in\left(t_{1}, t_{n}\right]
$$

where the local functions $\ell_{k}(t)$, defined on $\left(t_{k-1}, t_{k+1}\right)$, are scalar-, vector- or pointvalued. The ERBS Hermite interpolation properties (see Theorem 2.4 in [9]) states that an ERBS function interpolates the values and all existing derivatives of its local functions in their associated knots.

\subsection{Knot insertion}

Knot insertion is the process of inserting new knots into an existing knot vector and compute new coefficients for the splines which are non-zero in the affected knot intervals. This way the new and finer spline space contains the original coarser spline space. We mention here the Oslo algorithm [2] by Cohen, Lyche and Riesenfeld, which is appropriate when inserting more than a few knots at a time, and provide Boehm's method [1], to insert one knot at a time, in the following lemma:

Lemma 2.1. (Boehm's method). Let $\hat{\mathbf{t}}=\left(\hat{t}_{j}\right)_{j=1}^{n+d+1}$ be a given knot vector and let $\mathbf{t}=\left(t_{i}\right)_{i=1}^{n+d+2}$ be the knot vector obtained by inserting a knot $z$ in $\hat{\mathbf{t}}$ in the interval $\left[\hat{t}_{k}, \hat{t}_{k+1}\right]$. If

$$
f=\sum_{j=1}^{n} \hat{c}_{j} B_{j, d, \hat{\mathbf{t}}}(t)=\sum_{i=1}^{n+1} c_{i} B_{i, d, \mathbf{t}}(t),
$$

where $B_{j, d, \hat{\mathbf{t}}}(t)$ is the $j$ th $B$-spline of degree $d$ on the knot vector $\hat{\mathbf{t}}$, defined as

$$
B_{j, d, \hat{\mathbf{t}}}=\frac{t-\hat{t}_{j}}{\hat{t}_{j+d}-\hat{t}_{j}} B_{j, d-1, \hat{\mathbf{t}}}(t)+\frac{\hat{t}_{j+d+1}-t}{\hat{t}_{j+d+1}-\hat{t}_{j+1}} B_{j+1, d-1, \hat{\mathbf{t}}}(t),
$$


for all real numbers $t$, with

$$
B_{j, 0, \hat{\mathbf{t}}}(t)=\left\{\begin{array}{lc}
1, & \text { if } \quad \hat{t}_{j} \leq t \leq \hat{t}_{j+1} \\
0, & \text { otherwise }
\end{array}\right.
$$

then $\left(c_{i}\right)_{i=1}^{n+d+1}$ can be expressed in terms of $\left(\hat{c}_{j}\right)_{j=1}^{n}$ through the formulas

$$
c_{i}= \begin{cases}\hat{c}_{i}, & \text { if } 1 \leq i \leq k-d ; \\ \frac{z-\hat{t}_{i}}{\hat{t}_{i+d}-\hat{t}_{i}} \hat{c}_{i}+\frac{\hat{t}_{i+d}-z}{\hat{t}_{i+d}-\hat{t}_{i}} \hat{c}_{i-1}, & \text { if } k-d+1 \leq i \leq k ; \\ \hat{c}_{i-1}, & \text { if } k+1 \leq i \leq n+1 .\end{cases}
$$

For details on the topic of knot insertion we refer to [10].

\section{LOCAL REFINEMENT OF ERBS CURVES}

Given an existing ERBS curve, as defined in eq. (2), where the local functions $\ell_{k}(t)$ are vector-valued curves. Suppose we generate extra local curves to increase the flexibility whilst shaping the ERBS curve. The extra local curves can be blended with the original ERBS curve. We discuss in brief the blending construction in eq. (2) before we change the topic to knot insertion.

\subsection{The ERBS blending construction}

Let us consider eq. (2) on the interval $\left(t_{k}, t_{k+1}\right)$. It follows from the ERBS minimal support property that the only non-zero ERBS on that interval are $B_{k}(t)$ and $B_{k+1}(t)$. But since $B_{k}(t)+B_{k+1}(t)=1$, due to the partition of unity property, $B_{k+1}(t)$ can be expressed as $1-B_{k}(t)$. We can clearly see that the ERBS function is a blending of two local functions inside the knot interval:

$$
\begin{aligned}
f(t) & =\ell_{k}(t) B_{k}(t)+\ell_{k+1}(t)\left(1-B_{k}(t)\right) \\
& =\ell_{k+1}(t)+\left(\ell_{k}(t)-\ell_{k+1}(t)\right) B_{k}(t),
\end{aligned}
$$

when $t_{k}<t<t_{k+1}$. By utilizing the fact that $B_{k}\left(t_{k}\right)=1$, we write

$$
f(t)= \begin{cases}\ell_{k}\left(t_{k}\right), & \text { if } t=t_{k} \\ \ell_{k+1}(t)+\left(\ell_{k}(t)-\ell_{k+1}(t)\right) B_{k}(t), & \text { if } t_{k}<t<t_{k+1}\end{cases}
$$

Deriving eq. (8) at $t=t_{k}, k=1, \ldots, n$ yields the ERBS Hermite interpolation properties:

$$
D^{j} f\left(t_{k}\right)=D^{j} \ell_{k}\left(t_{k}\right), \quad \text { for } k=1, \ldots, n \text { and } j=0,1,2, \ldots
$$

Equation (9) shows that at $t=t_{k}, k=1, \ldots, n$, all derivatives of the ERBS curve are equal to the respective derivatives of the local curve. 

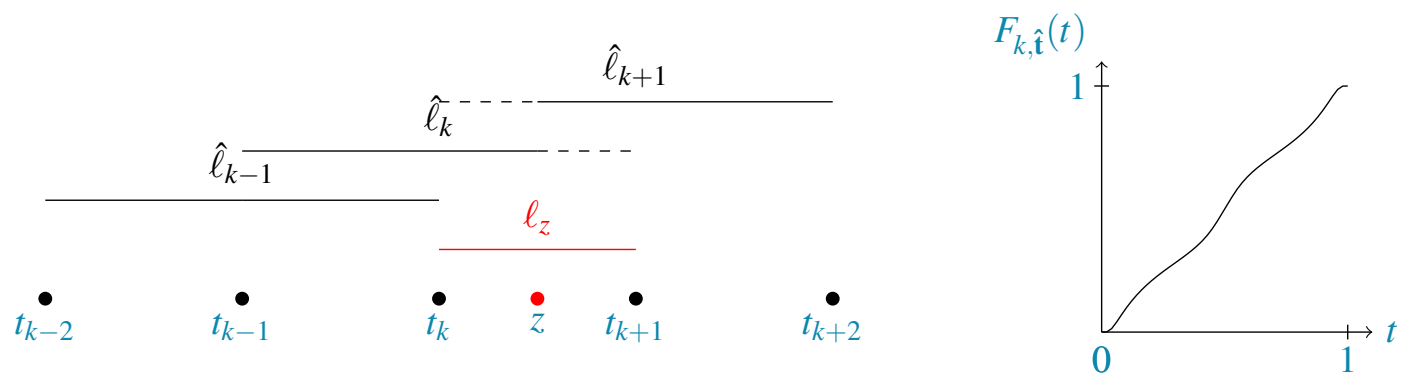

FIGURE 1. Left: Support of the local curves $\left(\ell_{i}\right)_{i=k-1}^{k+1}$ on the knot interval $\left(t_{k-1}, t_{k+1}\right)$. Dashed segments indicate parts of the local curves $\ell_{k}$ and $\ell_{k+1}$ which are not in use after inserting the knot $z$, with associated local curve $\ell_{z}$, between $t_{k}$ and $t_{k+1}$. Right: A plot of $F_{k, \hat{\mathbf{t}}}(t)$ in eq. (12) with $\hat{t}_{k}=0, z=0.5$ and $\hat{t}_{k+1}=1$.

\subsection{Knot insertion on ERBS curves}

The local curve for a new knot, inserted at an existing inner knot, can be obtained by constructing a copy of the existing knot's local curve. This is using the property that ERBS curves interpolate their local curves in their central knots. We will not discuss multiple knots further here, but focus on inserting new knots between existing knots.

Since each local curve $\ell_{k}(t)$ is supported on $\left(t_{k-1}, t_{k+1}\right)$ (see fig. 1), it follows that if we modify $\ell_{k}(t)$ to compensate for a new knot inserted in $\left(t_{k}, t_{k+1}\right)$, the change will influence the ERBS blending on the whole interval $\left(t_{k-1}, t_{k+1}\right)$. It not hard to imagine that such a change would cause a chain reaction invoked by adjusting $\ell_{k-1}(t)$, to compensate for the change of $\ell_{k}(t)$, which in turn triggers a need to adjust $\ell_{k-2}(t)$, and so on. We therefore apply the restriction of not changing any of the existing local curves in order to achieve local refinement.

In the following theorem, constrained by the mentioned restriction, we use Boehm's method (see lemma 2.1) to express the new local functions after knot insertion in terms of the existing:

Theorem 3.1. (Knot insertion on ERBS) Let $\hat{\mathbf{t}}=\left(\hat{t}_{j}\right)_{j=0}^{n+1}$ be a given knot vector and let $\mathbf{t}=\left(t_{i}\right)_{i=0}^{i=n+2}$ be the knot vector obtained by inserting a knot $z$ in $\hat{\mathbf{t}}$ in the interval $\left(\hat{t}_{k}, \hat{t}_{k+1}\right)$. If

$$
f(t)=\sum_{j=1}^{n} \hat{\ell}_{j}(t) B_{j, \hat{\mathbf{t}}}(t)=\sum_{i=1}^{n+1} \ell_{i}(t) B_{i, \mathbf{t}}(t),
$$

where $B_{j, \hat{\mathbf{t}}}(t)$ is the $j$ th ERBS on the knot vector $\hat{\mathbf{t}}$, defined as in eq. (1), then $\left(\ell_{i}(t)\right)_{i=1}^{n+2}$ can be expressed in terms of $\left(\hat{\ell}_{j}(t)\right)_{j=1}^{n+1}$ through the formulas

$$
\ell_{i}(t)= \begin{cases}\hat{\ell}_{i}(t), & \text { if } 1 \leq i<k, \\ \hat{\ell}_{i}(t) \circ w_{k}(t) & \text { if } i=k, \\ \hat{\ell}_{k}(t)+F_{k, \hat{\mathbf{t}}}(t)\left(\hat{\ell}_{k+1}(t)-\hat{\ell}_{k}(t)\right) & \text { if } i=k+1, \\ \hat{\ell}_{-1}(t) \circ w_{k+1}(t) & \text { if } i=k+2, \\ \hat{\ell}_{i-1}(t), & \text { if } k+2<i \leq n+1,\end{cases}
$$


where

$$
F_{k, \hat{\mathbf{t}}}(t)=\left\{\begin{array}{cl}
\frac{B \circ \hat{\omega}_{k}(t)}{B \circ \omega_{k}(t)} & \text { if } \hat{t}_{k}<t \leq z, \\
\frac{B \circ \hat{\omega}_{k}(t)-B \circ \omega_{k+1}(t)}{1-B \circ \omega_{k+1}(t)} & \text { if } z<t<\hat{t}_{k+1},
\end{array}\right.
$$

with $B(t)$ equal to the first half of the ERBS basis, as defined in the first part of eq. (1), on the interval $[0,1]$ and $\hat{\omega}_{k}(t)=\frac{t-\hat{t}_{k}}{\hat{t}_{k+1}-\hat{t}_{k}}, \omega_{k}(t)=\frac{t-\hat{t}_{k}}{z-\hat{t}_{k}}, \omega_{k+1}(t)=\frac{t-z}{\hat{t}_{k+1}-z}$ are affine functions mapping the parameter value $t$ within the new knot intervals to $[0,1]$, and the affine functions $w_{k}(t)=\frac{t-\hat{t}_{k-1}}{z-\hat{t}_{k-1}}$ and $w_{k+1}(t)=\frac{t-z}{t_{k+2}-z}$ are re-parameterizing the local functions, $\hat{\ell}_{k}(t)$ and $\hat{\ell}_{k+1}(t)$ respectively, to the new knot vector $\mathbf{t}$.

Proof. We look at the first two and the last two formulas in eq. (11). Observe that for $j<=k$ we have $\hat{t}_{j}=t_{j}$. For $i<k$ it follows that $\ell_{i}(t)=\hat{\ell}_{i}(t)$. When $i=k, \ell_{i}(t)=$ $\hat{\ell}_{i}(t) \circ w_{k}(t)$, since $\hat{\ell}_{i}(t)$ must be re-parameterized to the domain $\left(\hat{t}_{k-1}, z\right)$ on the new knot vector $\mathbf{t}$. Similarly, we have $t_{i}=\hat{t}_{i-1}$ for $i>k+1$. So $\ell_{i}(t)=\hat{\ell}_{i-1}(t)$ for such values of $i$, except when $i=k+2$, then $\ell_{i}(t)$ must be re-parameterized to the domain $\left(z, \hat{t}_{k+2}\right)$, hence, $\ell_{i}(t)=\hat{\ell}_{i-1}(t) \circ w_{k+1}(t)$.

Next, we discuss the case when $i=k+1$. As noted in eq. (7), the only non-zero ERBS on the interval $\left(\hat{t}_{k}, \hat{t}_{k+1}\right)$ are $B_{k, \hat{\mathbf{t}}}$ and $B_{k+1, \hat{\mathbf{t}}}$. Using the symmetry property of the ERBS basis function we rephrase eq. (7) to express the ERBS curve on the interval $\left(\hat{t}_{k}, \hat{t}_{k+1}\right)$ :

$$
\begin{aligned}
f(t) & =\hat{\ell}_{k}(t) B \circ\left(1-\hat{\omega}_{k}(t)\right)+\hat{\ell}_{k+1}(t) B \circ \hat{\omega}_{k}(t) \\
& =\hat{\ell}_{k}(t)+B \circ \hat{\omega}_{k}(t)\left(\hat{\ell}_{k+1}(t)-\hat{\ell}_{k}(t)\right)
\end{aligned}
$$

After knot insertion, the interval $\left(\hat{t}_{k}, \hat{t}_{k+1}\right)$ is divided into two new intervals; $\left(\hat{t}_{k}, z\right)$ and $\left(z, \hat{t}_{k+1}\right)$. The ERBS function in eq. (13) expressed on the new knot vector $\mathbf{t}$ is then

$$
f(t)= \begin{cases}\hat{\ell}_{k}(t)+B \circ \omega_{k}(t)\left(\ell_{z}(t)-\hat{\ell}_{k}(t)\right) & \text { if } \quad \hat{t}_{k}<t \leq z, \\ \ell_{z}(t)+B \circ \omega_{k+1}(t)\left(\hat{\ell}_{k+1}(t)-\ell_{z}(t)\right) & \text { if } \quad z<t<\hat{t}_{k+1} .\end{cases}
$$

The local function $\ell_{z}(t)$ associated with $z$ is found by solving the equation where the left hand side is given by eq. (13) and the right hand side is eq. (14):

$$
\ell_{z}(t)= \begin{cases}\hat{\ell}_{k}(t) \circ \omega_{k}(t)+\frac{B \circ \hat{\omega}_{k}(t)}{B \circ \omega_{k}(t)}\left(\hat{\ell}_{k+1}(t)-\hat{\ell}_{k}(t)\right) & \text { if } \quad \hat{t}_{k}<t \leq z, \\ \hat{\ell}_{k}(t)+\frac{B \circ \hat{\omega}_{k}(t)-B \circ \omega_{k+1}(t)}{1-B \circ \omega_{k+1}(t)}\left(\hat{\ell}_{k+1}(t)-\hat{\ell}_{k}(t)\right) & \text { if } \quad z<t<\hat{t}_{k+1} .\end{cases}
$$

A slight re-formulation of eq. (15) and exploiting eq. (12) leads to the middle formula in eq. (11).

We observe eq. (15) and conclude that the local function $\ell_{z}(t)$, associated with the new knot $z$, is represented through knot insertion in terms of the (original) local functions $\hat{\ell}_{k}(t)$ and $\hat{\ell}_{k+1}(t)$ and the rational function $F_{k, \hat{\mathbf{t}}}(t)$. The rational form is different from the original construction. $F_{k, \hat{\mathbf{t}}}(t)$ is different in the intervals $\left(\hat{t}_{k}, z\right)$ and $\left(z, \hat{t}_{k+1}\right)$.

We see from theorem 3.1 that $\omega_{k}(z)=1$. The first part of eq. (14) shows that $f(z)=$ $\ell_{z}(z)$. For reference, see the Hermite interpolation properties in eqs. (7) and (8). Since $\lim _{t \rightarrow z^{+}} \omega_{k+1}(t)=0, \lim _{t \rightarrow z^{-}} f(t)=\ell_{z}(t)$. This shows that eq. (12) is continuous on $\left(\hat{t}_{k}, \hat{t}_{k+1}\right)$. 
Both $\hat{\omega}_{k}(t)$ and $\omega_{k}(t)$ tend to zero as $t$ approaches $\hat{t}_{k}$ from above. But $\hat{\omega}_{k}(t)$ tends to zero faster than $\omega_{k}(t)$, since $z<t_{k+1}$, hence,

$$
\lim _{t \rightarrow \hat{t}_{k}^{+}} F_{k, \hat{\mathbf{t}}}(t)=\lim _{t \rightarrow \hat{t}_{k}} \frac{0}{B \circ \omega_{k}(t)}=0 .
$$

On the other hand, when $t$ is approaching $\hat{t}_{k+1}$ from below, both $\hat{\omega}_{k}(t)$ and $\omega_{k+1}(t)$ tend to one. But, since $\hat{t}_{k}<z, \hat{\omega}_{k}(t)$ tends to one faster than $\omega_{k+1}(t)$. Thus,

$$
\lim _{t \rightarrow \hat{t}_{k+1}-} F_{k, \hat{\mathbf{t}}}(t)=\lim _{t \rightarrow \hat{t}_{k+1}-} \frac{1-B \circ \omega_{k+1}(t)}{1-B \circ \omega_{k+1}(t)}=1 .
$$

A plot of $F_{k, \hat{\mathbf{t}}}(t)$, as defined in eq. (12), is provided in fig. 1.

Our final note here is that the observations on the limits of $F_{k, \hat{\mathbf{t}}}(t)$ as $t$ goes towards $\hat{t}_{k}$ and $\hat{t}_{k+1}$ shows that the middle formula in eq. (11), on the form of eq. (13), satisfies the ERBS Hermite interpolation properties in eqs. (7) to (9).

\subsection{Approximation of local functions}

As we have seen in the previous section, generating a local curve for a new knot inserted into an existing knot vector has a solution which involves a rational function. In practical applications the local functions are usually polynomials, such as Bézier curves, Hermite curves or circle arcs. It is therefore interesting to investigate methods to generate local curves of different brands and how well they approximate the original ERBS. We propose the following strategies to generate local curves:

I Use a truncated Taylor expansion of the original ERBS curve in the new knot. This is, due to the ERBS Hermite interpolation properties (see eqs. (7) to (9)), equivalent to evaluating the original ERBS curve given the new knot as its parametric value.

II Construct a new local curve, of the same brand as the existing local curves, which Hermite interpolates the value and all existing derivatives of the ERBS in the new knot.

III Construct a new local curve, of the same brand as the existing local curves, by performing a least squares approximation of the local curve specified by the middle formula in eq. (11) of theorem 3.1.

IV Adjust the coefficients of the local curve, obtained by any of the two previous methods, to obtain a better geometric approximation of the global ERBS curve.

As an example we consider a closed parametric ERBS curve, which approximates a circle with radius $r=10$ and parametric value $t \in[0,2 \pi)$, using four quadratic Bézier local curves on the knot vector $\mathbf{t}=\left\{\frac{3 \pi}{2}, 0, \frac{\pi}{2}, \pi, \frac{3 \pi}{2}, 0\right\}$. Table 1 shows error measurements for the different methods to generate local curves for the new knot $z=3.5$. We measure the deviation using norms presented in [9]; a max norm

$$
L^{\infty}(f-g)=\max _{t \in\left[t_{1}, t_{n+1}\right]}|f(t)-g(t)|,
$$


TABLE 1. Error measurements for different kinds of local curves when inserting one new knot on an ERBS approximation of a circle using four quadratic Bézier local curves.

\begin{tabular}{lrrrrr}
\hline & I & II & II + IV & III & III + IV \\
\hline$L^{2}(f-g)$ & 0.075 & 0.080 & 0.12 & 0.071 & 0.032 \\
$L^{\infty}(f-g)$ & 0.29 & 0.26 & 0.45 & 0.25 & 0.14 \\
$L_{G}^{2}(f-g)$ & 0.049 & 0.046 & 0.0078 & 0.026 & 0.0069 \\
$L_{G}^{\infty}(f-g)$ & 0.22 & 0.17 & 0.033 & 0.097 & 0.032 \\
\hline
\end{tabular}

to measure the guaranteed maximum deviation, and an $L^{2}$ norm

$$
L^{2}(f-g)=\sqrt{\frac{1}{t_{n+1}-t_{1}} \int_{t_{1}}^{t_{n+1}}|f(t)-g(t)|^{2} d t}
$$

to investigate the "quadratic" mean deviation. These two norms take the parameterization into consideration, thus, they are used to measure the "mathematical" deviation.

Another error measure, proposed in [9], which only refers to the geometric shape and not to the speed of the parameterization, using a non-symmetric version of the Hausdorff distance, for measuring the result, using a geometric version of a metric related to a max norm,

$$
L_{G}^{\infty}(f-g)=\max _{t \in\left[t_{1}, t_{n+1}\right]}\left|f(t)-C_{g}(f(t))\right|,
$$

where $C_{g}(\mathbf{p})$ refers to the closest point on a curve $f$ from a point $\mathbf{p}$. A metric related to the $L^{2}$ norm is constructed in [9] by the following:

$$
L_{G}^{2}(f-g)=\sqrt{\frac{\int_{t_{n}}^{t_{n+1}}\left|f(t)-C_{g}(f(t))\right|^{2}|D f(t)| d t}{\int_{t_{1}}^{t_{n+1}}|D f(t)| d t}} .
$$

The last method (IV) above is achieved here by a rather naïve, iterative algorithm which translates or dilates the coefficients of the Bézier curve in small steps; first along the line which passes through the first and the last coefficients, then along a line which passes through one of the "inner" coefficients and its closest point on the first line, until it reaches a stop criterion when the approximation error does not improve.

\section{CONCLUDING REMARKS}

Local refinement of ERBS curves, in terms of knot insertion, constrained by not altering any of the existing local curves, provides a rational function as the new coefficient.

We propose a few methods to approximate the local curves associated with the inserted knots. This is of interest in applications where it is desirable to use a homogeneous set of local curves. The error is restricted within the support intervals of the new local curves. 
Methods I and II shows near similar performance but the geometric shape of the global curve is different. Method III provides an increase in geometric deviation. Invoking IV shows that it is possible to improve the global geometric approximation error by altering the new local curve. But the mathematical deviation may increase, and the refined ERBS curve may not interpolate the original in the new knot.

As topics for future work we suggest investigating least squares approximation with respect to the global approximation error, knot insertion on the generalized set of ERBS basis functions (GERBS [11]), or even Sigmoid functions. It would also be interesting to investigate convergence when the value of the inserted knot becomes arbitrarily close to an existing knot.

A different approach to investigate local refinement in terms of knot insertion could be considering Taylor expansions as local coefficients rather than the polynomial functions considered here.

\section{ACKNOWLEDGMENTS}

The work described in this paper is a part of "Dreamworld", a joint research project between Funcom and Narvik University College, funded by the Norwegian Research Council under the Verdikt program with grant agreement No. 201511.

\section{REFERENCES}

1. W. Boehm, "Inserting New Knots into B-spline Curves," , Journal of Computer Aided Design 12, 199-201 (1980).

2. E. Cohen, T. Lyche, and R. Riesenfeld, "Discrete B-Splines and Subdivision Techniques in ComputerAided Geometric Design and Computer Graphics," , Computer Graphics and Image Processing 14, 87-111 (1980).

3. T. W. Sederberg, J. Zheng, A. Bakenov, and A. Nasri, "T-splines and T-NURCCs," in ACM SIGGRAPH 2003 Papers, SIGGRAPH '03, ACM, New York, NY, USA, 2003, pp. 477-484, ISBN 1-58113-709-5.

4. T. Dokken, T. Lyche, and K. F. Pettersen, "Polynomial splines over locally refined box-partitions," , Computer Aided Geometric Design 30, 331 - 356 (2013), ISSN 0167-8396.

5. E. Catmull, and J. Clark, "Recursively generated B-spline surfaces on arbitrary topological meshes,", Journal of Computer Aided Design 10, 350-355 (1978).

6. L. L. Schumaker, and T. Sorokina, "Smooth Macro-Elements on Powell-Sabin-12 Splits,", Mathematics of Computation 75, 711-726 (2006).

7. A. Lakså, B. Bang, and L. T. Dechevsky, "Exploring Expo-Rational B-splines for Curves and Surfaces," in Mathematical methods for Curves and Surfaces, edited by M. Dæhlen, K. Mørken, and L. Schumaker, Nashboro Press, 2005, pp. 253-262.

8. L. T. Dechevsky, A. Lakså, and B. Bang, "Expo-Rational B-Splines," , International Journal of Pure and Applied Mathematics 27, 319-362 (2006).

9. A. Lakså, Basic properties of Expo-Rational B-splines and practical use in Computer Aided Geometric Design, Ph.D. thesis, University of Oslo (2007).

10. R. N. Goldman, and T. Lyche, Knot Insertion and Deletion Algorithms for B-Spline Curves and Surfaces, Geometric Design Publications, SIAM, 3600 University City Science Center, Philadelphia, PA 19104-2688, 1993.

11. L. T. Dechevsky, B. Bang, and A. Lakså, "Generalized Expo-Rational B-Splines," , Int. J. Pure Appl. Math. 57, 833-872 (2009). 